\title{
Ferroelectric Poling of Methylammonium Lead lodide Thin Films
}

\author{
Holger Röhm, Tobias Leonhard, Michael J. Hoffmann, and Alexander Colsmann*
}

\begin{abstract}
Seemingly contradictory reports on polar domains and their origin have surrounded the controversial discussion about the ferroelectricity of the methyl ammonium lead iodide $\left(\mathrm{MAPbl}_{3}\right.$ ) thin films that are commonly employed in perovskite solar cells. In this work, microscopic modulations of the polar domain patterns upon application of an electric poling field are correlated with macroscopic changes to the currents through the $\mathrm{MAPbl}_{3}$ layer. Piezoresponse force microscopy is used to monitor the widening, narrowing, generation or extinction of polar domains, as well as shifts of the domain walls at room temperature under an in-plane electric poling field that is applied between two laterally organized electrodes. This poling leads to a net polarization of individual grains and the thin film itself. Macroscopically, this net polarization results in a persistent shift of the diode characteristics that is measured across the channel between the electrodes. Both the modulation of the polar domains upon electric poling and the concurrent persistent shift of the electric currents through the device are the unambiguous hallmarks of ferroelectricity, which demonstrate that $\mathrm{MAPb}_{3}$ is a ferroelectric semiconductor.
\end{abstract}

\section{Introduction}

Solar cells comprising methylammonium lead iodide $\left(\mathrm{MAPbI}_{3}\right)$ perovskites or derivatives thereof respond to electronic stimuli with a combination of many optoelectronic, ionic and mechanical effects. Understanding the underlying processes within the perovskite crystal lattice is pivotal to the design of measurement routines and the interpretation of data. Ferroelectricity is one of the often proposed properties of $\mathrm{MAPbI}_{3}$ in order to explain the charge carrier dynamics in solar cells, such as the

Dr. H. Röhm, T. Leonhard, Dr. A. Colsmann

Karlsruhe Institute of Technology

Light Technology Institute (LTI)

Engesserstrasse 13, 76131 Karlsruhe, Germany

E-mail: alexander.colsmann@kit.edu

Dr. H. Röhm, T. Leonhard, Prof. M. J. Hoffmann, Dr. A. Colsmann

Karlsruhe Institute of Technology

Material Research Center for Energy Systems (MZE)

Strasse am Forum 7, 76131 Karlsruhe, Germany

Prof. M. J. Hoffmann

Karlsruhe Institute of Technology

Institute for Applied Materials-Ceramic Materials and Technologies (IAM)

Haid-und-Neu-Strasse 7, 76131 Karlsruhe, Germany

The ORCID identification number(s) for the author(s) of this article can be found under https://doi.org/10.1002/adfm.201908657.

(C) 2019 The Authors. Published by WILEY-VCH Verlag GmbH \& Co. KGaA, Weinheim. This is an open access article under the terms of the Creative Commons Attribution License, which permits use, distribution and reproduction in any medium, provided the original work is properly cited.

DOI: 10.1002/adfm.201908657 low non-radiative recombination losses and the hysteresis in current density-voltage $(J-V)$ curves of solar cells. ${ }^{[1-3]}$ To date, the scientific community has not yet reached a consensus on the existence and the possible implications of ferroelectricity in $\mathrm{MAPbI}_{3}$ or other organic metal-halide $(\mathrm{OMH})$ perovskites. Besides ferroelectricity, ${ }^{[4]}$ ionic charge accumulation, ${ }^{[9,10]}$ ferroelasticity, ${ }^{[10-12]}$ and piezoelectricity ${ }^{[4,9]}$ have been proposed as the origin of dynamic electronic and mechanical changes in $\mathrm{OMH}$ samples. Early simulative work on $\mathrm{MAPbI}_{3}$ solar cells predicted a beneficial role of ferroelectric domains on the charge carrier extraction and recombination properties in photovoltaic devices..$^{[1,2]}$

The experimental hallmark of ferroelectricity is the polarizability of the crystal by an externally applied electrical field. Microscopically, ferroelectricity shows in the spontaneous formation of alternating polar domains which can be shaped (poled) under the electrical field. Such polar domains within crystals were observed via correlative atomic force microscopy (AFM) techniques. ${ }^{[6,7,13]}$ Other reports observed electrostriction or even macroscopic poling in $\mathrm{MAPbI}_{3}$, but concluded that ionic processes produce these effects, and hence they discarded ferroelectricity since classical hysteresis curves could not be obtained. ${ }^{9,14,15]}$

Macroscopically, classical ferroelectricity shows in a polarization hysteresis upon repetitive forward and backward sweeping of an external poling bias. Beyond a characteristic electric field strength (coercive E-field), the polarization saturates and stabilizes (remnant total polarization). ${ }^{[16]}$

Unfortunately, because of its higher conductivity over typical insulating ceramic ferroelectrics, the application of an electric field drives notable currents through $\mathrm{MAPbI}_{3}$, which can damage the sample and hence often impedes the direct proof or disproof of ferroelectricity. ${ }^{[5,6,17]}$ This is why, to date, claims of ferroelectric switching in $\mathrm{MAPbI}_{3}$ were often inconclusive, and sometimes microscopic and macroscopic observations of polarization were assigned to ionic charge accumulation and modulation of defects at interfaces. ${ }^{[9,10,14,15]}$ Some earlier reports succeeded in obtaining polarization curves, for example at low temperatures, ${ }^{[5,17]}$ but the high conductivity of the sample makes it very challenging to unambiguously differentiate between leakage and poling currents.

To proof ferroelectric switching of $\mathrm{MAPbI}_{3}$ thin films, we investigated the effects of rather weak electric fields on the perovskite layer, as sample damages can be avoided under moderate biases. On a time scale of several minutes, ferroelectric switching occurs in $\mathrm{MAPbI}_{3}$ thin films, which results 


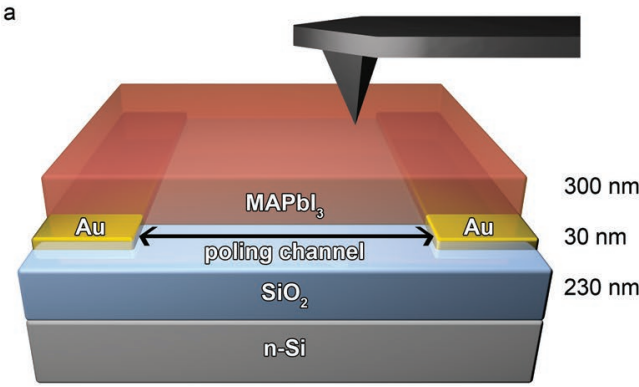



Figure 1. a) Layout of the sample architecture used for poling experiments. An insulating $\mathrm{SiO}_{2}$ layer between $\mathrm{MAPbl}_{3}$ and the n-doped $\mathrm{Si}$ substrate allows the application of a lateral electric field between the gold electrodes across the poling channel $(2.5,5$, or $10 \mu \mathrm{m})$. The conductive substrate serves as a counter-electrode to the scanning tip during PFM measurements. b) Top-view optical microscope image of the $\mathrm{MAPbl}_{3}$ layer atop interdigitated gold electrodes (here the channel width is $10 \mu \mathrm{m}$ ).

in changes of the polar domains on the nanometer scale that we observed by piezoresponse force microscopy (PFM). The so-created electric built-in field (remnant polarization) leads to directional conductivity of the perovskite layer, which is switchable over more than two orders of magnitude and persists for more than half an hour.

\section{Sample Design}

Earlier attempts (including our own) to electrically pole $\mathrm{MAPbI}_{3}$ employed a common solar cell architecture, i.e., an out-of-plane (vertical) electric field applied across a layer stack, which often led to a destruction of the perovskite layer. Recently, we demonstrated that large grains of $\mathrm{MAPbI}_{3}$ thin films exhibit vastly predominant in-plane (lateral) polarization. ${ }^{[18]}$ The energy, and hence the electric field, which is required to change the polarization of ferroelectric materials, is much lower if the field is applied collinear to the direction of polarization. ${ }^{[19]}$ This is why we have deliberately opted for the sample architecture in Figure $\mathbf{1}$ that is commonly used for bottom-gated thin-film transistors and that allows in-plane poling of the polycrystalline $\mathrm{MAPbI}_{3}$ layer in between two laterally organized electrodes.

Therefore, we deposited a perovskite thin film atop a $\mathrm{SiO}_{2}-$ insulated $\mathrm{n}$-Si wafer with prestructured $\mathrm{Au}$ electrodes forming channels of $2.5,5$, or $10 \mu \mathrm{m}$ in width. The two gold electrodes were used to apply lateral poling fields across the perovskite layers within the channel. We used the two-step deposition process for the $\mathrm{MAPbI}_{3}$ layer and the subsequent solvent-vapor annealing that we described in our previous reports on polar domains in perovskite solar cells. ${ }^{[6,18]}$ Importantly, this sample architecture also allows the direct monitoring of changes in the $\mathrm{MAPbI}_{3}$ microstructure upon poling since the $\mathrm{MAPbI}_{3}$ layer is not concealed by a counter electrode as it would be the case in a vertical poling geometry. The conductive $\mathrm{n}$-Si wafer serves as a counter-electrode to the AFM tip in the subsequent PFM characterization of the sample. We note that all fabrication steps and measurements in this work were performed in a glovebox under $\mathrm{N}_{2}$ atmosphere to exclude any ambient influences on the poling experiments and on the degradation of the samples. Even after several weeks of storage in $\mathrm{N}_{2}$ atmosphere, we observed no changes to the domain patterns of our samples.
We deliberately opted for a perovskite layer thickness of $300 \mathrm{~nm}$ in order to best represent the $\mathrm{MAPbI}_{3}$ layers that are typically employed in solar cells.

\section{Observation of Polar Domains}

Figure 2a depicts the surface topography of a representative MAPbI ${ }_{3}$ layer. Even though the $\mathrm{SiO}_{2}$ beneath provided a different surface than the poly(3,4-ethylenedioxythiophene):p olystyrenesulfonate (PEDOT:PSS) that we employed in our previous reports, grain sizes and topographies are very similar. As shown in Figure 2b, we measured the local piezoresponse of the perovskite grains by lateral PFM (LPFM) and found self-organized parallel stripes of alternating polarity. In the literature, the intricacies of PFM measurements of the soft, semiconducting and ionic $\mathrm{MAPbI}_{3}$ are discussed heavily, ${ }^{[10,20,21]}$ mostly converging toward two interpretations: i) the domain surfaces are charged, e.g., by ions, modulated by different mechanical moduli of the domains ${ }^{[9,10]}$ and ii) the domains exhibit alternating intrinsic polarization. ${ }^{[4-8]}$ However, the following three additional observations allow to rule out the former explanation: First, in accordance with our earlier work $^{[18]}$ and as exemplified in the Figure S1 in the Supporting Information, the vast majority of large grains in our samples did not show piezoelectric contrast in vertical PFM (VPFM) but only in LPFM. Notably, surface charges should, by means of electrostatic interaction, rather produce a contrast in VPFM, but less so in LPFM, independent of the type of charge. Second, the vastly predominant (110) texture of the annealed $\mathrm{MAPbI}_{3}$ layers and the concomitant in-plane confinement of the $c$-axis of the unit cell throughout the alternating domains, ${ }^{[18]}$ for symmetry reasons, should not produce any charge or ionic contrast at the sample surface. Third, the LPFM measurements produce a $180^{\circ}$ phase contrast between neighboring domains as depicted in Figure 2c. For a better visualization of the phase contrast, Figure $2 \mathrm{~d}$ represents the LPFM phase along the red bar in Figure 2c, and Figure 2e shows a phase histogram of the sample. Altogether, the LPFM/VPFM anisotropy, the symmetry of the sample surface and the distinct LPFM $180^{\circ}$ phase contrast let us conclude that the origin of the domain pattern indeed stems from an in-plane polarization of the domains rather than from ionic surface charges. 

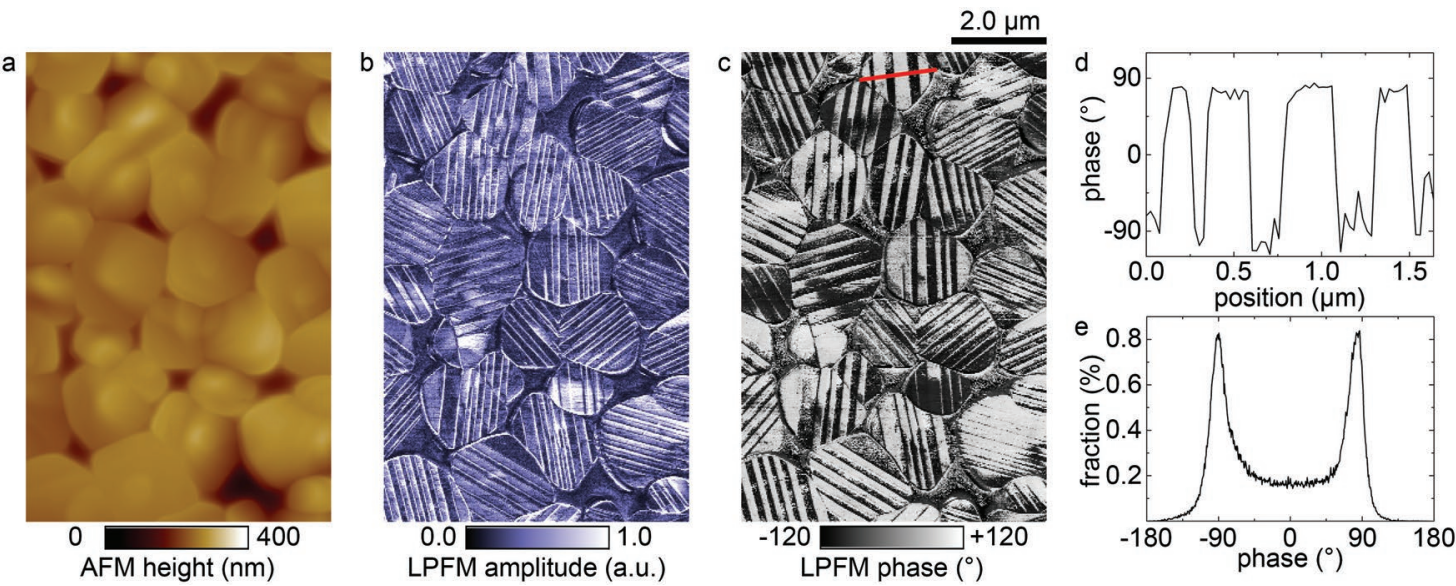

Figure 2. a) The AFM topography of a typical sample shows large, flat grains of $\mathrm{MAPbl}_{3}$. b) LPFM reveals parallel stripes of alternating polarization within the grains. c) Neighboring grains exhibit a phase contrast of $180^{\circ}$. d) Representative phase contrast along the red bar in (c). e) Phase histogram of the phase image $(c)$.

Since the vast majority of domains in our samples are polarized in-plane, we henceforth only show and discuss LPFM data. We note that the polarization directions and domain sizes may be different if bulk-MAPbI 3 is investigated (in contrast to thin films).

\section{Nanoscale Observations of DC Poling}

$\mathrm{MAPbI}_{3}$ shows distinct spontaneous alternating polarization in PFM, which alone is often considered a fingerprint of ferroelectricity. ${ }^{[22]}$ Yet, so far, ferroelectric switching has been hampered by its much higher conductivity compared to typical other classical ferroelectrics such as $\mathrm{BaTiO}_{3}$ or lead zirconate titanate (PZT). ${ }^{[23-25]}$ In order to gently switch the polarization without driving damaging currents through the perovskite layer, we applied a moderate in-plane electric field over a period of
10-30 min, a strategy that has been used successfully on other semiconducting ferroelectrics. ${ }^{[26-28]}$ The PFM images in Figure 3 depict the effects of lateral DC poling on the domain patterns.

Before poling, the $\mathrm{MAPbI}_{3}$ grains in Figure 3a exhibit parallel, $90 \mathrm{~nm}$ wide domains of alternating polarization. On many grains, the domain walls form $45^{\circ}$ angles with the grain boundaries. This domain pattern that has formed after continuous sample annealing for $1 \mathrm{~h}$, very likely represents a stable energetic optimum. Then the grain boundaries follow along energetically favorable crystal planes (e.g., (100) and (010)) approaching the equilibrium shape of the crystal which leads to square-shaped grains. This is in agreement with our previous observation that the polarization direction coincides with the $c$-axis of the perovskite unit cell and that the polarization directions of two neighboring domains form a $90^{\circ}$ angle. ${ }^{[18]}$ Accordingly, the domain walls form angles of $45^{\circ}$ with the polarization directions.

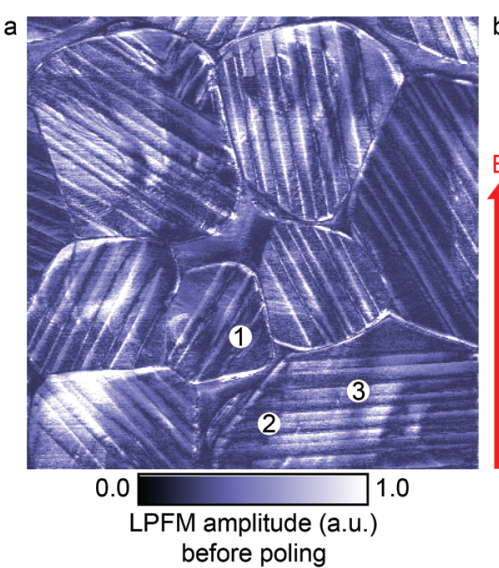

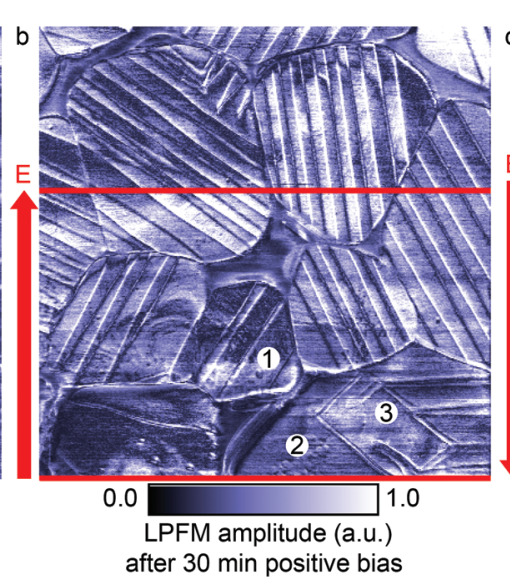

Figure 3. a) Before poling, the sample exhibits highly ordered domains with alternating polarization. Most grain boundaries are likely to show the equilibrium shape originating from the tetragonal unit cell, forming an angle of $45^{\circ}$ with the domain walls. b) After $\mathrm{DC}$ poling $\left(E=+2 \mathrm{~V} \mu \mathrm{m}^{-1}\right)$ over the channel (2.5 $\mu \mathrm{m}$, marked in red) some domains widen (position 1), and larger domains of unidirectional polarization occur (position 2). Domain walls which are oriented $45^{\circ}$ to the previously parallel domain walls appear (positions 2 and 3). c) After subsequent DC poling in the opposite direction, the distribution of domains becomes more asymmetric (position 1), and a previously poled domain decreases in size (position 3). The top area of all three images which is located atop one of the electrodes, and hence outside of the channel, remains unaffected by the electric poling field. 
Figure $3 \mathrm{~b}$ shows that, upon applying a moderate electric field of $E=+2 \mathrm{~V} \mathrm{\mu m}^{-1}$ for $30 \mathrm{~min}$, the domain patterns change drastically allowing the direct observation of the ferroelectric effect on the nanoscale. The area and direction of the poling field is indicated in red. For example, at position 1, three domains were narrowed and the position of the domain walls changed. At the same time, the complementary domains with alternate polarization expanded. Position 2 shows a large domain replacing the alternating domain pattern previously observed on the pristine sample. This large domain exhibits domain walls which are oriented $45^{\circ}$ relative to the domain walls before poling (position 3). This finding may indicate the emergence of a different type of domain walls, where the polarization directions of two neighboring domains form a $180^{\circ}$ angle. After application of an electric poling field in opposite direction $\left(E=-2 \mathrm{~V} \mu \mathrm{m}^{-1}\right)$, further changes to the domain pattern become visible in Figure $3 \mathrm{c}$. For example, the large domain at position 2 increases in size, while the domain at position 3 shrinks. Importantly, the domains in the area outside of the poling channel that was not exposed to the poling field (top parts of Figure $3 \mathrm{a}-\mathrm{c}$ ), remained unchanged, excluding dissipative warming of the sample as the origin of the observed changes. But even on those grains that were exposed to the poling field, many domains and domain walls remained unchanged. These pinning effects within grains and at grain boundaries are typical for ferroelectric thin-film samples, which are strongly influenced by mechanical stress and defect sites. ${ }^{[29]}$ Electric poling and the concurrent reorganization of domains change the mechanical stress as well as the distribution of screening charges within a grain. Some of these domains remain in their new state after cut off of the external poling field whereas others revert back to their original, energetically more stable and less strained state before poling. ${ }^{[30,31]}$

We propose that the mechanism of domain widening and narrowing during poling interacts with increasing or decreasing amounts of screening charges at domain walls, which stabilize ferroelectric domains and compensate mechanical stress generated by the switching process, as it was observed in thin films of classical ferroelectrics. ${ }^{[32,33]}$ An electric field with a component that coincides with the polarization direction of a domain will decrease screening charges and therefore widen the domain, while an electric field in opposite direction will enhance the screening field and decrease the domain width.

Taking into account the orientation of the AFM cantilever relative to the sample during the scan furthermore lets us determine the direction of polarization of individual domains. Figure $4 \mathrm{a}, \mathrm{b}$ depicts LPFM micrographs of two different positions within the poling channel of the sample before and after

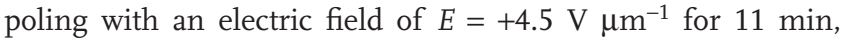
again displaying domain widening and narrowing. The AFM cantilever orientation during all scans in Figure $4 a, b, d, e$ is illustrated in Figure 4c. Figure 4d,e enlarges the white-framed areas of Figure $4 a, b$, highlighting grains where domains are narrowed (Figure 4d) or widened (Figure 4e). In LPFM, the inplane piezoresponse of the sample is detected via torsion of the cantilever as depicted in Figure 4f. Therefore, domains of high LPFM amplitude (bright color) must exhibit a larger polarization component perpendicular to the cantilever axis, and domains of low LPFM amplitude (dark color) must exhibit a larger polarization component parallel to the cantilever axis. ${ }^{[34]}$
This correlation is independent of the scanning direction of the AFM tip as long as the orientation of the cantilever relative to the sample is maintained.

Since all polarizations are oriented $45^{\circ}$ toward domain walls, and since the domains of low LPFM amplitude in Figure 4d,e must be polarized mainly in a parallel direction to the AFM cantilever and are narrowed by the electric poling field, we conclude the polarization pattern that is indicated with white and gray arrows. We note that, although the head-to-tail polarization vector configuration, which is depicted here, is energetically more favorable in common ferroelectrics with $90^{\circ}$ domain walls, ${ }^{[35,36]}$ we also observed grains where the phase contrast may be rather indicative of head-to-head configuration. For reasons of simplicity, here, we only discuss head-totail domain walls. Since the tetragonal unit cell of $\mathrm{MAPbI}_{3}$ is longer in $c$ - than in $a$ - and $b$-directions, $\left(a=b, c / a=1.0045^{[37]}\right)$, the reduction of the total (integrated) polarization of the grain in direction of the white arrows due to poling must strain this particular grain along the direction of the gray arrows. This also results in an overall net polarization of the grain in the same direction. In comparison, Figure 4e shows domains polarized mainly parallel to the cantilever (white arrows) that widen after poling. Here, the poled grain must exhibit strain and an overall net polarization in the direction of the white arrows.

\section{Macroscopic Effects of Ferroelectric Poling}

Besides the microscopic modulation of domains and domain boundaries, the ferroelectric poling also shows macroscopically in current-voltage sweeps across the channels between the $\mathrm{Au}$ electrodes. Classical insulating ferroelectrics typically produce a current-voltage curve that is a combination of a rising poling current which saturates beyond the coercive voltage and a small leakage current. In thin films of semiconducting ferroelectrics such as $\mathrm{MAPbI}_{3}$, the leakage current dominates these characteristics, often concealing the current components that stem from ferroelectric poling. ${ }^{[16]}$ Often, the saturation regime is not reached in semiconducting ferroelectrics, since high currents through the semiconductor destroy the layer. To exclude interface effects at the electrodes as the origin of the poling characteristics, we deliberately investigated channels with widths of $2.5,5$, and $10 \mu \mathrm{m}$. For better comparability of the characteristics across different channel sizes, we discuss current versus electric field $(I-E)$ sweeps for all samples. The samples were kept in the dark during the measurements in order to avoid contributions of photogenerated charges. The experiments were again carried out in a $\mathrm{N}_{2}$-glovebox to avoid parasitic effects from oxygen or water contamination of the samples.

Figure 5a depicts an $I-E$ sweep over a channel width of $5 \mu \mathrm{m}$ on a representative sample before poling. The electric field is modulated between +2 and $-2 \mathrm{~V} \mathrm{\mu m}^{-1}$ at a constant speed of $0.16 \mathrm{~V}\left(\mu \mathrm{m}^{-1} \mathrm{~s}^{-1}\right)$ over a time of $t_{\text {sweep }}=50 \mathrm{~s}$. Beyond an onset electric field of $E_{\text {on }}= \pm 1.6 \mathrm{~V} \mu \mathrm{m}^{-1}$, the freshly prepared device shows Schottky-diode behavior with exponentially increasing conductivity and significant conductivity retention upon subsequent voltage decrease. The device response is symmetric for positive and negative biases. Devices with channel widths

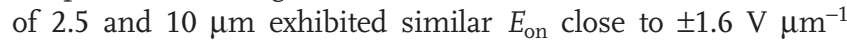




\section{Sample position 1}

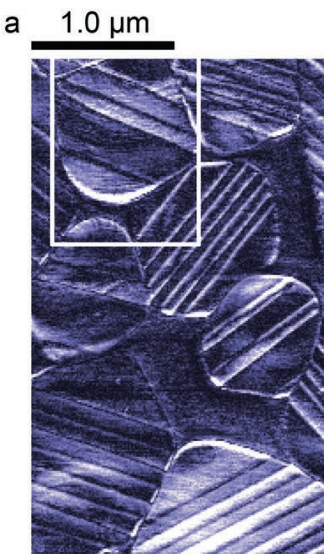

before poling

d $500 \mathrm{~nm}$

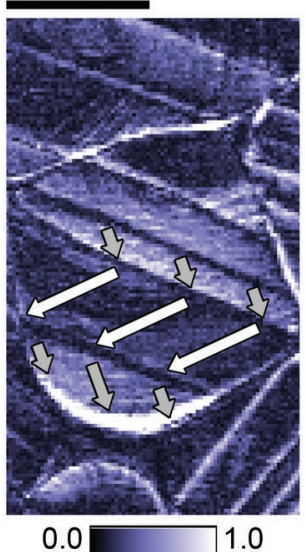

LPFM amplitude (a.u.)

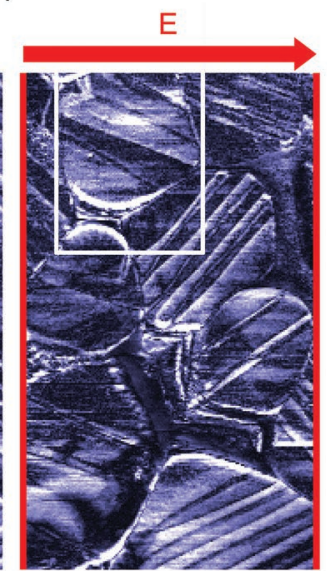

after poling



0.0

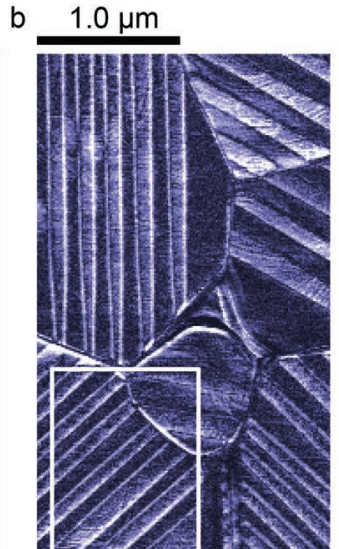

before poling e $500 \mathrm{~nm}$

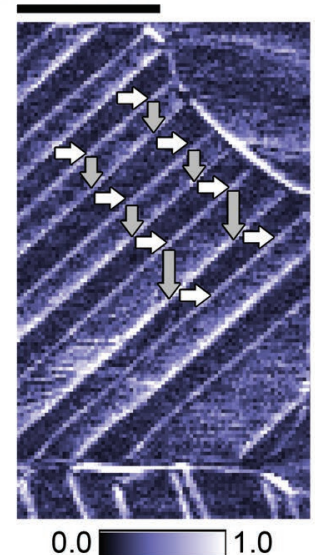

Sample position 2

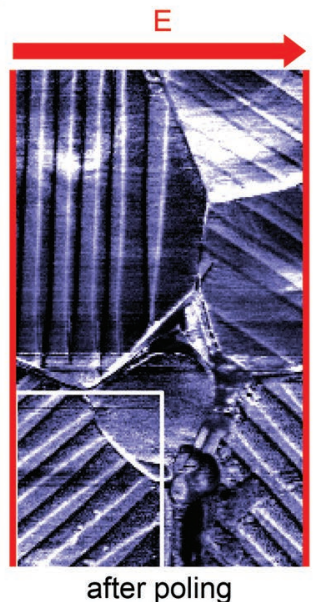

cantilever orientation during scan of images $a, b, d$ and $e$

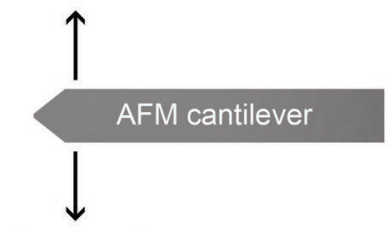

direction of in-plane sensitivity

\section{f}
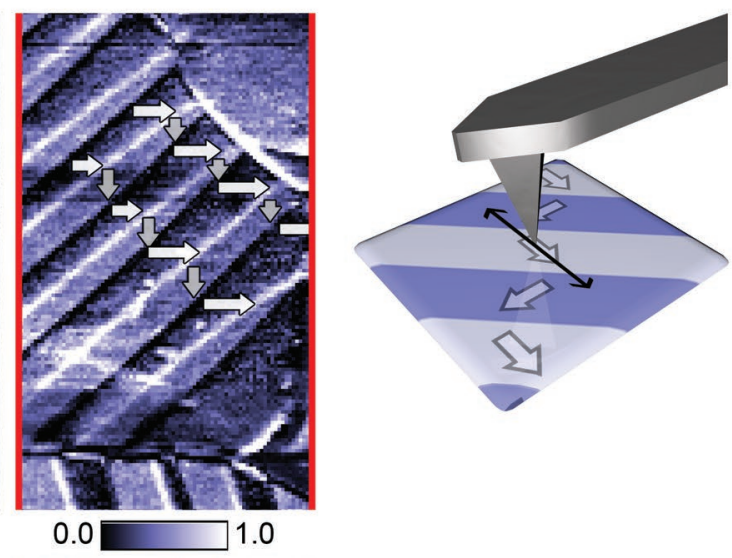

LPFM amplitude (a.u.) LPFM amplitude (a.u.)

Figure 4. a) LPFM amplitude of a MAPbl 3 thin film before (left) and after (right) poling. After poling with $E=+4.5 \mathrm{~V} \mu \mathrm{m}^{-1}$ for 11 min, the size and shape of domains change significantly. b) A second sample position shows similar changes after poling. c) A schematic top view of the AFM cantilever orientation during the scan illustrates the directions of LPFM sensitivity to the piezoresponse of the sample. d,e) The magnification of the white-framed areas in (a) and (b) exemplifies the widening and narrowing of domains upon poling. The gray and white arrows indicate the polarization direction of domains that was deduced from the correlation of the amplitude signal, the cantilever sensitivity direction and the direction of the poling field. The arrow length represents the projected width of each domain in polarization direction. f) Illustration of the interaction between the polarization direction of the sample and the torsion amplitude of the cantilever (black arrows) from which the LPFM data are derived.

(see Figure S2 in the Supporting Information). This independence of $E_{\text {on }}$ from the channel width lets us conclude that the $I-E$ characteristic is determined by bulk-properties of the polycrystalline $\mathrm{MAPbI}_{3}$ thin film rather than $\mathrm{Au} / \mathrm{MAPbI}_{3}$ interface effects.

In the next step, we applied a constant DC poling bias of $+2 \mathrm{~V} \mathrm{\mu m}^{-1}$ to the device for $600 \mathrm{~s}$, i.e., the maximum field that occurred during $I-E$ sweeping. During the first $10 \mathrm{~s}$ of poling, the sample current increased. After this initial period of increasing current, the current declined to a steady-state current of $120 \mathrm{nA}$ (Figure $5 \mathrm{~b}$ ). $120 \mathrm{~s}$ after completion of the $600 \mathrm{~s}$ poling period at $+2 \mathrm{~V} \mathrm{\mu m}^{-1}$, we recorded the $I-E$ sweep that is depicted in Figure 5c. As $E_{\text {on }}$ shifts to $+0.5 \mathrm{~V} \mathrm{\mu m}^{-1}$, we conclude the formation of a built-in field of approximately $1 \mathrm{~V} \mathrm{\mu m}^{-1}$. Under negative biases, the device showed excellent blocking (1 $\mathrm{nA}$ at $-2 \mathrm{~V} \mu \mathrm{m}^{-1}$ ). The changes of ferroelectric domains during DC poling as discussed in Figures 3 and 4 led to a net polarization within $\mathrm{MAPbI}_{3}$ grains as illustrated underneath each $I-E$ curve. This remnant net polarization after poling created the observed built-in field and, therefore, modulates the $I-E$ curves of the device. This effect remained largely stable over 45 min after poling and during multiple sweeps but showed some gradual changes such as increasing $E_{\text {on }}$ over time (Figure S3, Supporting Information). Finally, we poled the same device in reverse direction with a negative bias. As depicted in Figure $5 d$, applying a negative bias for several minutes, significantly enhanced the conductivity in backward direction. After $360 \mathrm{~s}$, a reverse steady-state current with a magnitude of $110 \mathrm{nA}$ is reached which is similar to the positive poling current before. Accordingly, the remnant polarization is inverted and so is the $I-E$ characteristic in Figure 5e, demonstrating sample poling in the opposite direction. We note that, in our experiment, individual $I-E$ sweeps with either positive or negative biases between 0 and $\pm 2 \mathrm{~V} \mathrm{\mu m}^{-1}$ did not result in a significant remnant poling of the sample, but multiple consecutive sweeps resulted in an 

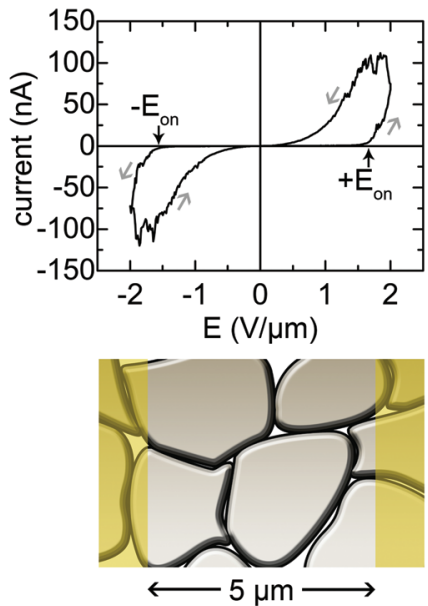

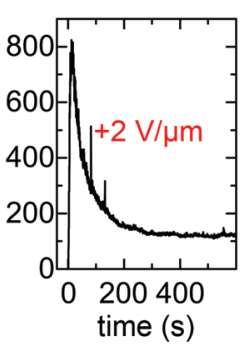

positive poling


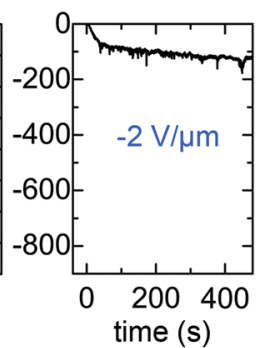

negative poling

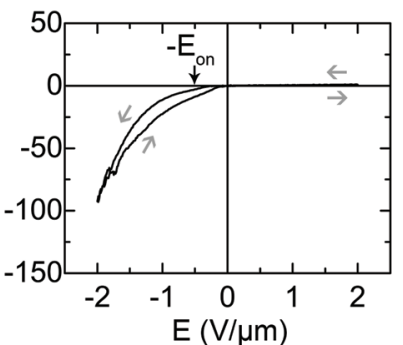

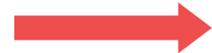

Figure 5. a) $I-E$ sweep of a representative $\mathrm{MAPbl}_{3}$ thin film before poling in between two laterally organized Au electrodes which form a channel of $5 \mu \mathrm{m}$ in width and $10 \mathrm{~mm}$ in length, exhibiting Schottky-diode behavior. At $E_{\mathrm{on}}= \pm 1.6 \mathrm{~V} \mu \mathrm{m}^{-1}$, the conductivity increases exponentially. Upon reversing

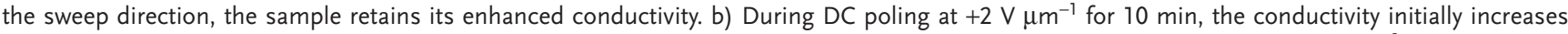
and then steadily decreases toward a steady-state current of $120 \mathrm{nA}$. c) After DC poling, the $I-E$ sweeps show an $E_{\text {on }}=+0.5 \mathrm{~V} \mu \mathrm{m}^{-1}$, and the device efficiently blocks charge carriers under reverse electric fields. d) A subsequent negative $D C$ poling bias of $-2 \mathrm{~V} \mu \mathrm{m}^{-1}$ increases the sample current from -1 to $-110 \mathrm{nA}$ within the first $6 \mathrm{~min}$. e) The $I-E$ sweep characteristic is inverted compared to poling under forward bias. Underneath each $I-E$ curve, the proposed formation of a net built-in field within the grains after poling is illustrated.

incremental polarization of the sample over time (Figure S4, Supporting Information), similar to DC poling. During symmetric $I-E$ sweeps as shown in Figure 5a,c,e, only minor poling can occur due to the short timeframe of $5 \mathrm{~s}$ during which the electric field exceeds $1.6 \mathrm{~V} \mu \mathrm{m}^{-1}$. Upon symmetric sweeping

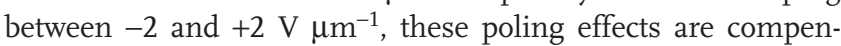
sated leaving no significant net polarization in the device.

\section{Origin of Poling Effects}

The ferroelectric domain switching on the nanoscale, as observed by PFM above, produces a net polarization of the individual grains and hence a lateral built-in field in the $\mathrm{MAPbI}_{3}$ thin films, which shifts the macroscopic Schottky-diode characteristic. The Schottky-diode characteristic itself can be attributed to a combination of the semiconductor-metal interface effects, that are mostly independent of the channel width, and energetic barriers of grain boundaries and screening charges at ferroelectric domain walls, as was reported for other ferroelectric materials such as $\mathrm{BiFeO}_{3}$ and PZT. ${ }^{[36,38-41]}$ Pantel et al. and Hong et al. demonstrated that the energy of the Schottky barrier in their samples coincided with the minimum energy of ferroelectric switching. ${ }^{[39,40]}$ Likewise, we propose that the Schottky-diode onset $E_{\text {on }}$ in $\mathrm{MAPbI}_{3}$ coincides with the first shifting of domain walls, which is hampered by domain wall pinning and ionic charges at grain boundaries in the $\mathrm{MAPbI}_{3}$ thin films at lower electric fields as illustrated in Figure $\mathbf{6}$.

We observed sharp current peaks in both $I-E$ sweeps and DC poling currents that might originate from single events of pinning-effect mediated poling in small volumes of the sample during a creeping poling process (Figures S2-S4, Supporting Information) as it was observed in some classical ferroelectrics too. ${ }^{[42,43]}$ Electric fields stronger than $2 \mathrm{~V} \mathrm{\mu m}^{-1}$ may trigger faster poling during single sweeps but, in our experiments, sometimes resulted in irreversible damage to the sample due to high currents (break-through). Any significant contribution of ionic drift to the poling current can be excluded by considering the total amount of $\mathrm{MAPbI}_{3}$ unit cells in the $5 \mu \mathrm{m}$ wide channel. A purely ionic sample current of the observed magnitude (Figure $5 b$ ) would require the removal of one ion from every primitive perovskite unit cell within the channel within the

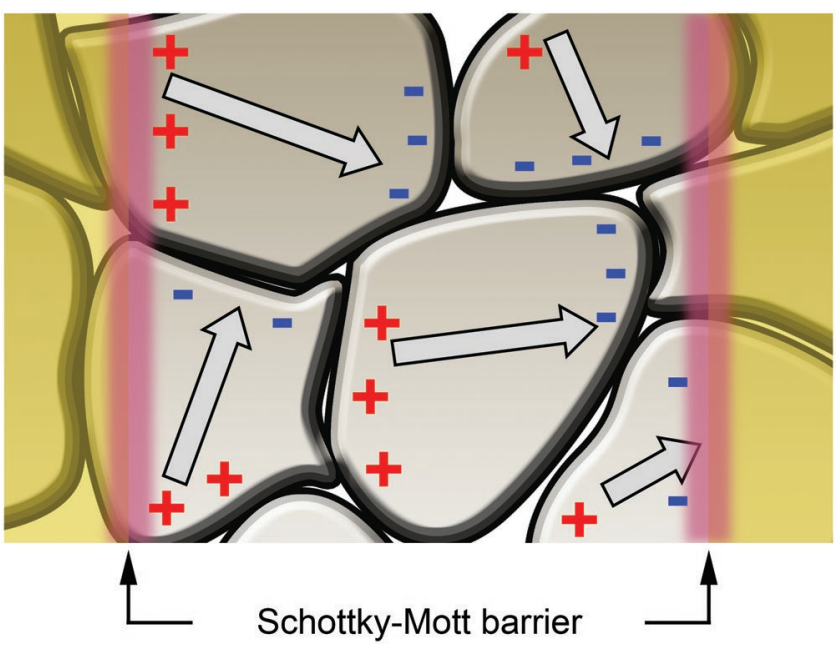

Figure 6. Illustration of the proposed interplay of ferroelectric polarization of grains, ionic and electronic screening charges, grain-boundary effects and metal-semiconductor interfaces that altogether lead to a complex electronic behavior of $\mathrm{MAPbl}_{3}$ thin films. Perovskite grains can form a Schottky contact with the gold electrodes (red highlight), leading to a diode onset during $I-E$ characterization. After the poling process, the lateral ferroelectric remnant polarization of individual grains leads to screening charges at grain boundaries and an overall net sample polarization in the poling channel. 
first $10 \mathrm{~s}$ of the DC current, which would result in a complete disintegration of the perovskite layer (calculation in Table S1 in the Supporting Information). Yet, the high ionic conductivity of $\mathrm{MAPbI}_{3}$ likely influences the $I-E$ sweeps and the poling process itself. We speculate that the screening charges at the ferroelectric domain walls or grain boundaries are mostly ionic in the dark, since, even without illumination, these charge carriers are present and mobile in $\mathrm{MAPbI}_{3}$. Upon generation of free electrons and holes under illumination, these charge carriers may replace and set free the ionic screening charges that can then subsequently modulate defects in the crystal lattice. Birkhold et al. previously reported ionic charging and PL quenching near electrodes on comparable timescales which indicates that slow poling fields are likely to influence the defect densities in $\mathrm{MAPbI}_{3}$ due to ionic movement. ${ }^{[44]}$ Other reports described similar ionic charge build-up or even chemical interaction of ionic species with the electrode interface at high voltages. ${ }^{[45,46]}$

This coupling of ferroelectricity and ionic effects can be disentangled by distinguishing relaxation timescales in samples that exhibit ionic conductivity as the predominant contribution to their electronic response. ${ }^{[47]}$ However, in a sample that is both semiconducting and heavily governed by grain boundary effects, such as a $\mathrm{MAPbI}_{3}$ thin film, the interplay is even more complex.

In order to disentangle ferroelectric effects and ionic or intrinsic conductivity, we conducted poling experiments at $90{ }^{\circ} \mathrm{C}$, far above the Curie temperature of $55^{\circ} \mathrm{C}$, where $\mathrm{MAPbI}_{3}$ presents itself in the cubic non-ferroelectric phase. Notably, Vorpahl et al. reported the disappearance of the domain patterns already at lower temperatures of $65^{\circ} \mathrm{C}$. ${ }^{[7]}$ At $90{ }^{\circ} \mathrm{C}$, our samples showed significantly higher conductivity and a lower $E_{\text {on }}= \pm 0.8 \mathrm{~V} \mathrm{\mu m}^{-1}$ (Figure S5, Supporting Information). Since ferroelectric effects are unlikely to play a role at $90{ }^{\circ} \mathrm{C}$, the remaining energy barrier must stem from grain boundaries and electrode interfaces as has been reported before. ${ }^{[48,49]}$ Even at moderate biases of $0.4 \mathrm{~V} \mathrm{\mu m}^{-1}$, this barrier is reduced during DC poling, leaving a small asymmetric poling effect in subsequent $I-E$ sweeps. We infer that this minor non-ferroelectric polarization stems from ionic charge built-up and possibly changes to the semiconductor-metal interface which, however, is much smaller than the ferroelectric poling effect that we observe at room temperature.

The direction of polarization relative to the external poling field strongly influences whether or not a change of polarization can be achieved. Our samples are mostly polarized in-plane and hence are unlikely to be polarizable by an out-of-plane electric field perpendicular to the domain polarization. An out-of-plane electric field would therefore require a significantly higher poling field to switch the polarization direction, which in turn would drive higher currents through the sample. In solar cells, the electrodes and selective charge carrier transport layers would generate out-of-plane electrical fields with only moderate amplitudes. Hence, only solar cells comprising vertical polarization components of the ferroelectric domains are polarizable. On solar cells with predominant in-plane polarization, using the same deposition route as employed for the lateral poling experiments above, we observed no significant influence of DC poling, neither on the maximum-power-point-tracked solar cell efficiency nor on the $J-V$ curves (Figure S6, Supporting Information). Recently, Ebadi et al. reported on negative capacitance effects in a variety of perovskite solar cells based on dynamic changes of conductivity or charge injection. ${ }^{[50]}$ The unknown cause for this time-dependent effect may be attributed to ferroelectric poling as reported here.

\section{Conclusions}

We have demonstrated that ferroelectric poling of $\mathrm{MAPbI}_{3}$ can be achieved by application of an electric field. The semiconducting nature of $\mathrm{MAPbI}_{3}$ required the application of mod-

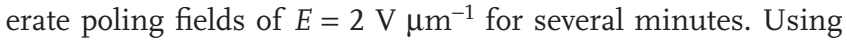
stronger poling fields and shorter periods of time produced high sample currents which destroyed the $\mathrm{MAPbI}_{3}$ layer. The observed changes to the nanoscale domain patterns upon poling led to a macroscopic remnant polarization of grains and layers that modulated the non-linear $I-E$ characteristic.

The complex time-dependent relationship of electric fields and sample currents hampered previous attempts to distinguish between ferroelectric poling currents, induced screening charges and interface effects. The electric poling itself was impeded in earlier studies by disregarding the polarization direction in $\mathrm{MAPbI}_{3}$ grains. Since our samples exhibited mostly in-plane polarization, electric poling required a lateral poling field, whereas all attempts to pole the device in a vertical poling field inevitably led to a destruction of the $\mathrm{MAPbI}_{3}$ layer. We speculate that vertically polarized domains, if they exist in a sample, can only be switched by vertical electric poling fields. Unfortunately, the switching of domains would then be concealed by the top electrode which makes the $\mathrm{MAPbI}_{3}$ layer inaccessible to PFM.

Importantly, the moderate poling fields applied herein have the same order of magnitude as the field between the electrodes of a perovskite solar cell at the maximum power point during illumination. As a consequence, we expect some self-induced poling to occur in $\mathrm{MAPbI}_{3}$ based solar cells which influences charge-carrier transport and extraction. For example, this selfpoling may occur during common light-soaking experiments that change the performance of perovskite solar cells under illumination for several minutes. And even during the recording of a common $J-V$ curve of a perovskite solar cell, the electric fields may be strong enough to introduce some degree of poling if vertically polarized domains exist. Hence, this poling effect may partly induce the $J-V$ hysteresis that is often observed in perovskite solar cells. If we incorporate our in-plane polarized $\mathrm{MAPbI}_{3}$ layers into solar cells, the hysteresis is almost negligible. While hysteresis in $\mathrm{MAPbI}_{3}$ solar cells are commonly attributed to ionic effects, our results suggest a contribution of ferroelectric poling in samples with out-of-plane polarization components.

Beyond perovskite solar cells, our experiments evidenced that even simple metal-MAPbI ${ }_{3}-$ metal architectures produce electric characteristics similar to a double Schottky diode which is programmable by preceding DC poling, such that the conductivity of the device in forward and backward direction can be reversibly adjusted over more than two orders of magnitude. This may enable a new class of cheap programmable electronic devices made from $\mathrm{OMH}$ perovskites. Yet, long term stability and reproducible characteristics are to be shown in order to enable their application. 


\section{Experimental Section}

Sample Preparation: All $\mathrm{MAPbl}_{3}$ samples used in this work were applied atop prestructured field-effect transistor substrates purchased from the Fraunhofer Institute for Photonic Microsystems: n-Si substrates $\left(n=3 \times 10^{17} \mathrm{~cm}^{-3}\right.$ near wafer surface) were thermally oxidized to form a $230 \mathrm{~nm}$ thick insulating layer of $\mathrm{SiO}_{2}$. Atop the $\mathrm{SiO}_{2}$ layer, $30 \mathrm{~nm}$ thick Au electrodes with $10 \mathrm{~nm}$ indium tin oxide adhesion layers underneath form 2.5, 5.0, and $10 \mu \mathrm{m}$ wide channels. Each channel is $10 \mathrm{~mm}$ long. Before perovskite layer deposition, the substrates were consecutively cleaned with acetone, isopropanol and $\mathrm{O}_{2}$ plasma (2 min). The perovskite layer was deposited in an inert $\mathrm{N}_{2}$ glovebox following a two-step process: $\mathrm{Pbl}_{2}$ (Sigma-Aldrich) was dissolved in dimethyl sulfoxide at $250 \mathrm{~g} \mathrm{~L}^{-1}$, filtered (PTFE, $0.2 \mu \mathrm{m}$ pore size), and mixed with 1-chloronaphtalene (100:1 v/v). $112.5 \mu \mathrm{L}$ of this formulation was added between a preheated doctor blade (Zehntner ZUA-2000, gap $70 \mu \mathrm{m}$ ) and the heated substrate (substrate width $45 \mathrm{~mm}$ ), before it was coated at $4 \mathrm{~mm} \mathrm{~s}^{-1}$ at $60^{\circ} \mathrm{C} .25 \mathrm{~s}$ after deposition, the wet-film was quenched with a stream of $\mathrm{N}_{2}$ gas for $10 \mathrm{~s}$. For the second deposition step, methylammonium iodide and methylammonium chloride (Lumtec, 9:1 w/w) were dissolved in anhydrous isopropanol at a total concentration of $40 \mathrm{~g} \mathrm{~L}^{-1}$. This solution was spin-cast atop the $\mathrm{Pbl}_{2}$ layer at 1100 RPM $\left(23 \mu \mathrm{L} \mathrm{cm}^{-2}\right)$. Immediately after spin-coating, the substrates were transferred to a hotplate at $100{ }^{\circ} \mathrm{C}$, covered by a Petri dish and annealed for $60 \mathrm{~min}$. For the PFM measurements, the $\mathrm{n}-\mathrm{Si}$ substrates (i.e., the counter electrodes) were contacted by removing the $\mathrm{SiO}_{2}$ insulation layer by scratching and subsequent application of silver paste in order to create a contact pad. All samples were kept in $\mathrm{N}_{2}$ atmosphere during fabrication, storage and all measurements in order to avoid degradation or any other influence by $\mathrm{O}_{2}$ or $\mathrm{H}_{2} \mathrm{O}$ on the samples' properties.

PFM Measurements: All AFM and PFM measurements were conducted inside an inert $\mathrm{N}_{2}$ glovebox on a Dimension Icon AFM (Bruker). Electrically conductive probes (SCM-PIC) with platin-iridium coated tips were used for all PFM measurements. A metal finger contact connected the previously prepared silver-ink pad of the n-Si substrate and the AFM measurement setup. In order to enhance the signal-tonoise ratio of the LPFM signal, an AC excitation voltage of $1.5 \mathrm{~V}$ was applied at $178-180 \mathrm{kHz}$, approximately $3 \mathrm{kHz}$ below the torsional contact resonance frequency of the cantilever. This signal enhancement is particularly important for PFM measurements of $\mathrm{MAPbl}_{3}$, where relatively low piezoelectric coefficients of about $5 \mathrm{pm} \mathrm{V}^{-1}$ were reported in earlier studies on single crystals. ${ }^{\left[{ }^{51,52]}\right.}$ Since, in thin films, clamping effects can lower the effective piezoelectric coefficient, it is expected to be even lower in the samples used in this work.

Since PFM measurements near contact resonance cannot be reliably calibrated, arbitrary units (a.u.) were used for LPFM and VPFM measurements in this manuscript. Typically, a setpoint of $70 \mathrm{~nm}$ was used, resulting in the application of a force of $14 \mathrm{nN}$ on the sample by the AFM tip. All PFM measurements in this work were adjusted to detect in-plane (LPFM) polarization. For a comparison of LPFM and VPFM on similar samples, Figure S1 (Supporting Information) and the previous work of the authors of this paper on the microstructure of $\mathrm{MAPbl}_{3}$ are referred. ${ }^{[18]}$ Since VPFM detects the deflection of the cantilever, it is prone to influences by electrostatic interaction between cantilever and sample as Collins et al. have recently reported. ${ }^{[53]}$ Similarly, Charrier et al. have shown that KPFM measurements on $\mathrm{Au}$ electrodes (similar to the work of this paper) can be influenced by the cantilever orientation relative to the interdigitated electrode structure. ${ }^{[54]}$ LPFM, however, detects the torsional movement of the cantilever caused by lateral movement of the sample and hence is unlikely to be influenced by vertical electrostatic interaction.

Electric Characterization and Poling: For I-E sweeps and DC poling, the Au-contact pads of the poling-channel electrodes were contacted by spring-suspended contact fingers inside an inert $\mathrm{N}_{2}$ glovebox. Both $I-E$ sweeps and DC poling current measurements were conducted on a Keithley 2450 Source Meter Unit. A scan rate of $0.16 \mathrm{~V}\left(\mu \mathrm{m}^{-1} \mathrm{~s}^{-1}\right)$ was used for all $I-E$ sweeps.

\section{Supporting Information}

Supporting Information is available from the Wiley Online Library or from the author.

\section{Acknowledgements}

The authors thank Manuel Hinterstein, Susanne Wagner, Alexander Schulz, Christian Sprau, and Uli Lemmer for fruitful discussions as well as Silvia Janietz (Fraunhofer IAP, Potsdam) for providing the prestructured transistor substrates. The work was supported by the Helmholtz Program Science and Technology of Nanosystems (STN) and the Landesgraduiertenförderung Baden-Württemberg.

\section{Conflict of Interest}

The authors declare no conflict of interest.

\section{Keywords}

electric poling, ferroelectricity, perovskite solar cells, piezoresponse force microscopy

Received: October 19, 2019

Published online:

[1] J. M. Frost, K. T. Butler, F. Brivio, C. H. Hendon, M. v. Schilfgaarde, A. Walsh, Nano Lett. 2014, 14, 2584.

[2] A. M. A. Leguy, J. M. Frost, A. P. McMahon, V. Garcia Sakai, W. Kockelmann, C. Law, X. Li, F. Foglia, A. Walsh, B. C. O'Regan, J. Nelson, J. T. Cabral, P. R. F. Barnes, Nat. Commun. 2015, 6, 7124.

[3] A. Pecchia, D. Gentilini, D. Rossi, M. Auf der Maur, A. Di Carlo, Nano Lett. 2016, 16, 988.

[4] Y.-J. Kim, T.-V. Dang, H.-J. Choi, B.-J. Park, J.-H. Eom, H.-A. Song, D. Seol, Y. Kim, S.-H. Shin, J. Nah, S.-G. Yoon, J. Mater. Chem. A 2016, 4, 756.

[5] Y. Rakita, O. Bar-Elli, E. Meirzadeh, H. Kaslasi, Y. Peleg, G. Hodes, I. Lubomirsky, D. Oron, D. Ehre, D. Cahen, Proc. Natl. Acad. Sci. USA 2017, 114, E5504.

[6] H. Röhm, T. Leonhard, M. J. Hoffmann, A. Colsmann, Energy Environ. Sci. 2017, 10, 950.

[7] S. M. Vorpahl, R. Giridharagopal, G. E. Eperon, I. M. Hermes, S. A. L. Weber, D. S. Ginger, ACS Appl. Energy Mater. 2018, 1, 1534.

[8] B. Chen, X. Zheng, M. Yang, Y. Zhou, S. Kundu, J. Shi, K. Zhu, S. Priya, Nano Energy 2015, 13, 582.

[9] Q. Dong, J. Song, Y. Fang, Y. Shao, S. Ducharme, J. Huang, Adv. Mater. 2016, 28, 2816.

[10] Y. Liu, L. Collins, R. Proksch, S. Kim, B. R. Watson, B. Doughty, T. R. Calhoun, M. Ahmadi, A. V. levlev, S. Jesse, S. T. Retterer, A. Belianinov, K. Xiao, J. Huang, B. G. Sumpter, S. V. Kalinin, B. Hu, O. S. Ovchinnikova, Nat. Mater. 2018, 17, 1013.

[11] I. M. Hermes, S. A. Bretschneider, V. W. Bergmann, D. Li, A. Klasen, J. Mars, W. Tremel, F. Laquai, H.-J. Butt, M. Mezger, R. Berger, B. J. Rodriguez, S. A. L. Weber, J. Phys. Chem. C 2016, 120, 5724.

[12] E. Strelcov, Q. Dong, T. Li, J. Chae, Y. Shao, Y. Deng, A. Gruverman, J. Huang, A. Centrone, Sci. Adv. 2017, 3, e1602165.

[13] H. Röhm, T. Leonhard, A. D. Schulz, S. Wagner, M. J. Hoffmann, A. Colsmann, Adv. Mater.31, 2019, 1806661.

[14] Z. Xiao, Y. Yuan, Y. Shao, Q. Wang, Q. Dong, C. Bi, P. Sharma, A. Gruverman, J. Huang, Nat. Mater. 2015, 14, 193. 
[15] B. Chen, T. Li, Q. Dong, E. Mosconi, J. Song, Z. Chen, Y. Deng, Y. Liu, S. Ducharme, A. Gruverman, F. De Angelis, J. Huang, Nat. Mater. 2018, 17, 1020

[16] B. Jaffe, W. R. Cook Jr., H. Jaffe, Piezoelectric Ceramics, Academic Press, London 1971

[17] Z. Fan, J. Xiao, K. Sun, L. Chen, Y. Hu, J. Ouyang, K. P. Ong, K. Zeng, J. Wang, J. Phys. Chem. Lett. 2015, 6, 1155.

[18] T. Leonhard, A. D. Schulz, H. Röhm, S. Wagner, F. J. Altermann, W. Rheinheimer, M. J. Hoffmann, A. Colsmann, Energy Technol. 2019, 7, 1800989.

[19] Y. A. Genenko, S. Zhukov, S. V. Yampolskii, J. Schütrumpf, R. Dittmer, W. Jo, H. Kungl, M. J. Hoffmann, H. v. Seggern, Adv. Funct. Mater. 2012, 22, 2058.

[20] A. D. Schulz, H. Röhm, T. Leonhard, S. Wagner, M. J. Hoffmann, A. Colsmann, Nat. Mater. 2019, 18, 1050

[21] Y. Liu, L. Collins, R. Proksch, S. Kim, B. R. Watson, B. Doughty, T. R. Calhoun, M. Ahmadi, A. V. levlev, S. Jesse, S. T. Retterer, A. Belianinov, K. Xiao, J. Huang, B. G. Sumpter, S. V. Kalinin, B. Hu, O. S. Ovchinnikova, Nat. Mater. 2019, 18, 1051

[22] J. Fousek, J. Appl. Phys. 1969, 40, 135

[23] C. Motta, F. El-Mellouhi, S. Sanvito, Sci. Rep. 2015, 5, 12746.

[24] S. S. Kumbhar, M. A. Mahadik, P. K. Chougule, V. S. Mohite, Y. M. Hunge, K. Y. Rajpure, A. V. Moholkar, C. H. Bhosale, Mater. Sci.-Pol. 2016, 33, 852.

[25] Y.-Y. Lee, L. Wu, C.-K. Liang, T.-S. Wu, Ferroelectrics 1993, 138, 11.

[26] S. Zhukov, S. Fedosov, J. Glaum, T. Granzow, Y. A. Genenko, H. v. Seggern, J. Appl. Phys. 2010, 108, 014105.

[27] S. Y. Wu, Ferroelectrics 1976, 11, 379.

[28] S. Wada, A. Seike, T. Tsurumi, Jpn. J. Appl. Phys. 2001, 40, 5690.

[29] E. L. Colla, D. V. Taylor, S. Hong, A. K. Tagantsev, K. No, N. Setter, Integr. Ferroelectr. 1998, 22, 237.

[30] Y. W. Li, X. L. Zhou, F. X. Li, J. Phys. D: Appl. Phys. 2010, 43, 175501.

[31] R. Xu, S. Liu, I. Grinberg, J. Karthik, A. R. Damodaran, A. M. Rappe, L. W. Martin, Nat. Mater. 2015, 14, 79.

[32] A. Gruverman, B. J. Rodriguez, R. J. Nemanich, A. I. Kingon, J. Appl. Phys. 2002, 92, 2734.

[33] H. Lu, X. Liu, J. D. Burton, C.-W. Bark, Y. Wang, Y. Zhang, D. J. Kim, A. Stamm, P. Lukashev, D. A. Felker, C. M. Folkman, P. Gao, M. S. Rzchowski, X. Q. Pan, C.-B. Eom, E. Y. Tsymbal, A. Gruverman, Adv. Mater. 2012, 24, 1209.

[34] E. Soergel, J. Phys. D: Appl. Phys. 2011, 44, 464003.
[35] E. A. Eliseev, A. N. Morozovska, G. S. Svechnikov, V. Gopalan, V. Y. Shur, Phys. Rev. B 2011, 83, 235313.

[36] D. Meier, J. Seidel, A. Cano, K. Delaney, Y. Kumagai, M. Mostovoy, N. A. Spaldin, R. Ramesh, M. Fiebig, Nat. Mater. 2012, 11, 284.

[37] Y. Dang, Y. Liu, Y. Sun, D. Yuan, X. Liu, W. Lu, G. Liu, H. Xia, X. Tao, CrystEngComm 2015, 17, 665.

[38] T. Choi, Y. Horibe, H. T. Yi, Y. J. Choi, W. Wu, S. W. Cheong, Nat. Mater. 2010, 9, 253

[39] D. Pantel, S. Goetze, D. Hesse, M. Alexe, ACS Nano 2011, 5, 6032

[40] S. Hong, T. Choi, J. H. Jeon, Y. Kim, H. Lee, H.-Y. Joo, I. Hwang, J.-S. Kim, S.-O. Kang, S. V. Kalinin, B. H. Park, Adv. Mater. 2013, 25, 2339.

[41] C. Wang, K.-j. Jina, Z.-t. Xu, L. Wang, C. Ge, H.-b. Lu, H.-z. Guo, M. He, G.-z. Yang, Appl. Phys. Lett. 2011, 98, 192901.

[42] Z. Gao, H. Zhang, Y. Liu, L. Wu, J. Yang, T. Zhang, H. Wang, X. Chen, G. Wang, H. He, Curr. Appl. Phys. 2017, 17, 495.

[43] M. Alexe, M. Ziese, D. Hesse, P. Esquinazi, K. Yamauchi, T. Fukushima, S. Picozzi, U. Gösele, Adv. Mater. 2009, 21, 4452.

[44] S. T. Birkhold, J. T. Precht, H. Liu, R. Giridharagopal, G. E. Eperon, L. Schmidt-Mende, X. Li, D. S. Ginger, ACS Energy Lett. 20183, 1279.

[45] I. M. Hermes, Y. Hou, V. W. Bergmann, C. J. Brabec, S. A. L. Weber, J. Phys. Chem. Lett. 2018, 9, 6249.

[46] J. Pospisil, A. Guerrero, O. Zmeskal, M. Weiter, J. J. Gallardo, J. Navas, G. Garcia-Belmonte, Adv. Funct. Mater.29, 2019, 1900881.

[47] A. N. Morozovska, E. A. Eliseev, N. V. Morozovsky, S. V. Kalinin, Phys. Rev. B 2017, 95, 195413.

[48] Y. Yuan, T. Li, Q. Wang, J. Xing, A. Gruverman, J. Huang, Sci. Adv. 2017, 3, e1602164.

[49] M. Stumpp, R. Ruess, J. Müßener, D. Schlettwein, Mater. Today Chem. 2017, 4, 97.

[50] F. Ebadi, N. Taghavinia, R. Mohammadpour, A. Hagfeldt, W. Tress, Nat. Commun. 2019, 10, 1574.

[51] Y.-J. Kim, T.-V. Dang, H.-J. Choi, B.-J. Park, J.-H. Eom, H.-A. Song, D. Seol, Y. Kim, S.-H. Shin, J. Nah, S.-G. Yoon, J. Mater. Chem. A 2015, 4, 756.

[52] D. Seol, G. S. Han, C. Bae, H. Shin, H. S. Jung, Y. Kim, J. Mater. Chem. A 2015, 3, 20352.

[53] L. Collins, Y. Liu, O. S. Ovchinnikova, R. Proksch, ACS Nano 2019, $13,8055$.

[54] D. S. H. Charrier, M. Kemerink, B. E. Smalbrugge, T. de Vries, R. A. J. Janssen, ACS Nano 2008, 2, 622. 